\title{
A mechanism of resource co-reservation in cloud computing system based on virtual resource
}

\author{
Jing $\mathrm{Li}^{1, \mathrm{a}}$ \\ ${ }^{I}$ Department of Mathematics and Information Engineering, Chongqing \\ University of Education, Chongqing 400067, China \\ ali_jing1@163.com \\ *Jing $L I$
}

\begin{abstract}
Resource reservation is an important part of the resource management in Large Distributed System such as cloud computing. An architectural framework for specifying and processing co-reservations in cloud computing environments is presented. Compared to other approaches, the co-reservation framework in this paper is more general. The concept of Virtual Resources is introduced that allows to compose resources by abstracting from their specific features for green computing. Virtual Resources enable advanced co-reservation with nested resource levels, resource aggregation, and transparent fault recovery.
\end{abstract}

Keywords: cloud computing, green computing, virtual resource, QoS.

\section{Introduction}

In order to guarantee the quality of service(QoS) in large distributed system such as cloud computing, resource reservation is a very effective approach[1]. In general, two types of resource reservations in computer networks can be distinguished: immediate reservations which are made in a just-in-time manner and advance reservations which allow to reserve resources a long time before they are actually used. Advance reservations are especially useful for cloud computing but also for a variety of other applications. In advance reservation environments, additional new services can be served such as malleable reservations which can lead to an increased QOS of the network. There are some experiment system of large distributed environment has adopt Advance reservations such as Globus[2] and so on.

The reservation requests of user generally include starting time, insisting time, resource types and reservation types, etc. Because the job on the cloud system is usually complicated, involving a lot of resource, when the resource is reserved, we can reserve more than one resource, which is called resource 
co-reservation. For example, interactive data analysis needs to access storage system possessing data replicas, make use of super computer to analyze data, make use of network to transfer data, and meanwhile utilize visualization equipment to communicate between human and machine. Ever resource in the above example requires quality of service. For the sake of that, resource co-reservation is a feasible approach.

Document[3] show that advance reservation may lead to resource fragment and the drop of network performance, putting forward a flexible reservation mechanism, can improve network performance effectively. The document compared four flexible reservation approaches, and the result shows, that some approaches add resource fragment, and some improve network performance. Despite of discussing performance issue, the document provides else the software framework of advance reservation management system.

Globus Architecture Reservation and Allocation(GARA) [2] is the first architecture that want to confirm the Grid QoS by means of resource reservation. It provides uniformed API of preserve process on various resource for users and applications.

Existing resource reservation approaches focus on the stability of reserved resource, but in the large distributed environment, resource is highly dynamic, which is the inherent character, and so that the above mentioned methods of resource reservation are limited.

This paper combines the resource reservation mechanism with the service-oriented framework, providing a new resource co-reservation mechanism in the large distributed environment, which constructs a visual resource model, shielding the dynamicity of resource, having the property of fault tolerance.

\section{Visual Resource Model}

\subsection{Hierarchical model of visual resource}

In the large distributed environment, solving the problem of resource sharing coordinately on dynamic and heterogeneous visual organizations requires highly controllability. Resource reservation is a kind of resource sharing too, a booking resource sharing. Existing resource reservation approaches have definitely defined that what is the resource, and who provides the resource, and who uses the resource, and when sharing the resource. While virtual resource shielding the resource provider and the physical resource itself, is a service-oriented resource reservation architecture. 
In applications, the construction of VR is transient, having specific demand and target, that is to say, constructing a VR aimed to some user's once resource reservation request. In a lot of cases, VR can be considered as the collection of resource which is geographic distributed, logically adjacent, and function complemental, showing the external characters of standard service interface.

In order to realize resource sharing and coordinate work in distributed heterogeneous network, we must solve the interactive operation issues. In other grid resource reserve mechanism, the goal is achieved by standardization of protocol architecture and realization of API and SDK.

(1)It can realize the abstraction of specific resource, covering the difference of resource's realization means.

(2)Different services with standard interface can be integrated to form higher services, and higher services themselves also have standard interface and call specification.

(3)It can realize consistent resource reservation approach crossover heterogeneous platforms.

(4)It can realize the separation of logical service instance and physical resource.

(5)It can map the service to the local running platform, realizing managing the remote services by local running platform.

Besides that, WSDL realize standard denotation of grid service, considering platform's diversity not any more. This method adopts the concept of encapsulation, and it is called Virtualization.

We provide the Visual Resource model is Hierarchical, which is shown an Figure1.

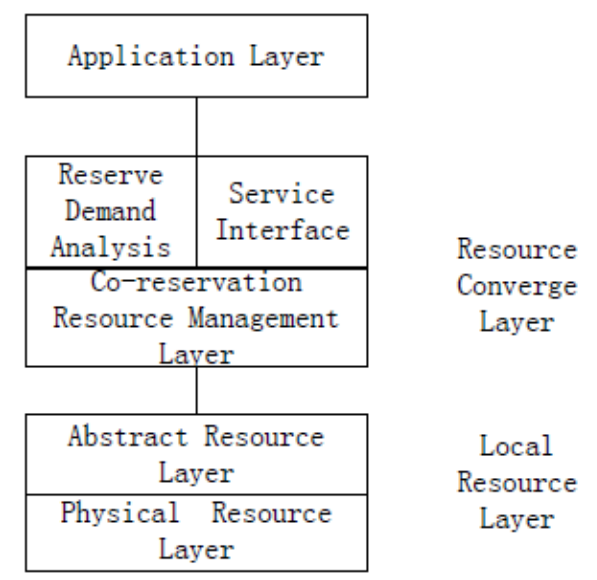

Fig. 1 Visual resource hierarchical model 
In order to achieve the solution of resource sharing and co-reservation in dynamic and multi-organization system, the resource reservation mechanism must be able to provide services on the base of a variety of local resource, and so that the architecture should firstly cover the difference of various resources, then realize the reservation of any single resource. On that basis, multiple resources co-reservation is accomplished, and at last many kinds of applications with a virtual resource are provided. These functions are brought into effect in sequence by the lays in the architecture respectively. The functions of the layers are described as follows.

Local resource layer: including physical resource layer and abstract resource layer. The physical resource layer realizes the access and control of local specific resource, providing resource reservation and sharing-access interface for higher layer. The abstract resource layer realize reservation of single resource, and the protocols are including security negotiation, initialization, detection, control, fee counting, and fee paying.

Resource converge layer: including resource co-reservation management layers, reserved resource analysis and service interfaces, providing analysis of users' reservation demand, detection of various resource's state, queue of backing resource, and standard service interfaces of various resource's co-reservation.

Application layer: including application programs of resource reservation in the system, by means of calling underlying API to get corresponding services.

Life cycle of visual resource starts with reserve request provided by specific application, then resource converge layer processes co-reserve of multi-resource, and it does end with the close of specific application. VR shows stable external property, being able to give assurance of quality to specific application. At the same time, it is dynamic inside, not limited by fixed resource provider, being able to combine new resource again continuously according to detection the state of various resource and queue of backing resource, giving up the old depressed resource, getting a dynamic balance, which is fitting in with the highly dynamics of grid. VR takes on the characteristics of fault tolerance and load balance whose resource state detection mechanism can find the error resource in time taking place with new backing resource, and also can find heavy-loaded resource that will be replaced with light-loaded resource.

\subsection{Definition of RESOURCE CO-RESERVATION}

We define the resource co-reservation $\mathrm{R}$ according to following parameters:
--ts: Reserve start time
--te: Reserve end time 
--sc: Reserve service type

--ri:The type of various resource, for example, computing resource, network resource, or memory resource

--c $(\mathrm{r}, \mathrm{t})$ :Capability function, returning the capability provided by resource $\mathrm{r}$ at moment $\mathrm{t}$

According to these concepts, we can define the demand of multi-resource co-reservation as follws:

$\mathrm{R}(\mathrm{ts}, \mathrm{te}, \mathrm{sc},\{(\mathrm{r} 1, \mathrm{c}(\mathrm{r} 1, \mathrm{t})), . .,(\mathrm{rn}, \mathrm{c}(\mathrm{rn}, \mathrm{t}))\})$

\section{Simulations}

We used simulations of a network with a power law link distribution PLOD[4] to validate our analytical results. The prototype system is programmed by java, and tested on Linux.

Experiment 1 Tolerance comparison

On the aspect of fault tolerance, we compared the reservation mechanism on the base of VR and fixed resource. Suppose in the system the type of resource is 50 , the total number of resource is from 64 to 4096, each resource being invalid with probability from $10 \%$ to $20 \%$. Resource reservation experiments are done with group of different resource number. Every group is tested 10 times, and the failure probability is figured out. The result is shown as Figure 1. The abscissa is the number of resource, and the ordinate is the failure probability of resource reservation.

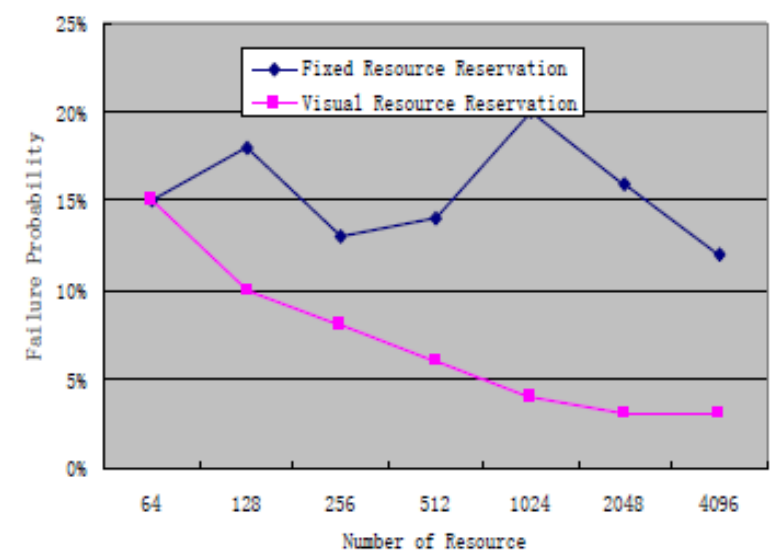

Fig. 2 Comparison of fault tolerance

The experiment result shows that the fault tolerance performance of resource preserve mechanism based on VR is better than based on fixed resource. The more amount of resource 
Experiment 2 Load balance comparison

Figure 3 shows the comparison result of system load balance taking on two approaches.

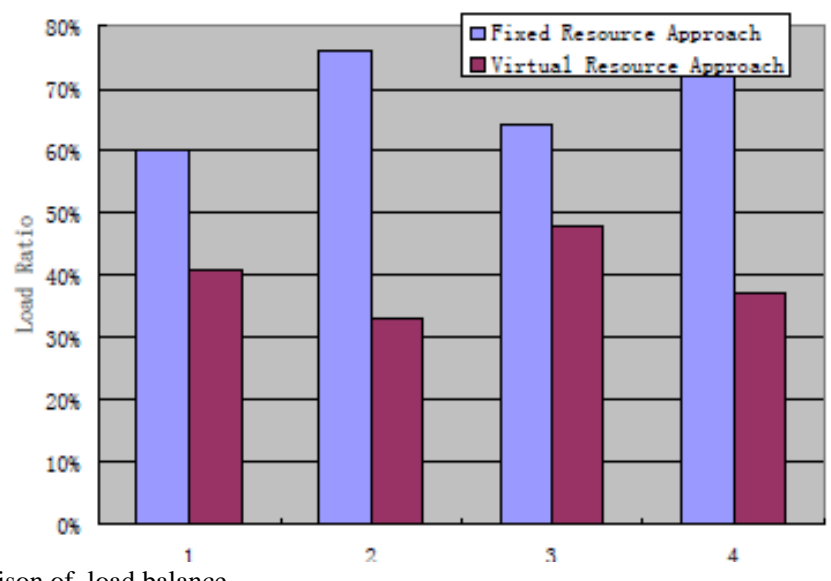

Fig.3 Comparison of load balance

In the figure, $\mathrm{X}$ axis denotes 4 times of experiment, $\mathrm{Y}$ axis denotes the ratio of resource request received by 10 nodes whose load is the most heavy, to the total request. From figure 3, by the means of fixed resource reservation, the resource is not well balanced, so that the user's request maybe can not be effectively processed. While by the means of virtual resource reservation, the resource is well balanced, and the selected resource is more effective.

\section{Conclusion}

In large distributed system, existing resource reservation mechanism is not flexible. We propose a mechanism of resource co-reservation based on visual resource model. In this model, we preserve resource from service-oriented perspective, providing service quality assurance for service consumers. Visual resource having the property of stability, dynamic and user-transparent fault tolerance, is a feasible resource reservation mechanism for green computing.

\section{References}

[1] I. Foster, C. Kesselman, C. Lee, R. Lindell, K. Nahrstedt, and A. Roy. A Distributed Resource Management Architecture that Supports Advance Reservations and Co-Allocation. Proceedings of the International Workshop on Quality of Service, 1999, 121-134. 
[2] http://www.globus.org

[3] Burchard L-O, Heiss H-U, De Rose C. Performance Issues of Bandwidth Reservations for Cloud Computing. Proceedings of 15th Symposium on Computer Architecture and High Performance Computing, 2013, 82-90.

[4] C Palmer, J Steffan. Generating network topologies that obey power law. In: Process of GLOBECOM. San Francisco, CA: IEEE, 2010, 434-438. 